\title{
GENERALIZED QUOTIENTS WITH APPLICATIONS IN ANALYSIS *
}

\author{
PIOTR MIKUSIŃSKI ${ }^{\dagger}$
}

\begin{abstract}
Starting from a nonempty set $X$ and a commutative semigroup $G$ acting on $X$ we construct a new space $\mathcal{B}(X, G)$ whose algebraic character is similar to a quotient field. The construction of the quotient field from an integral domain is a special case of our construction. Other interpretations of the construction include the space of Schwartz distributions of finite order, tempered distributions, Radon measures, and Boehmians.

In this paper we describe the construction of $\mathcal{B}(X, G)$, discuss some general properties of $\mathcal{B}(X, G)$, and present some applications of the construction.
\end{abstract}

1. Introduction. Rational numbers are usually defined as the field of quotients constructed from the ring of integers $\mathbb{Z}$. We would like to consider a somewhat different point of view. Let $\mathbb{N}$ denote the multiplicative semigroup of natural numbers acting on $\mathbb{Z}$ by multiplication. Then every $n \in \mathbb{N}$ is identified with an injective mapping $n: \mathbb{Z} \rightarrow \mathbb{Z}$. The set of rational numbers $\mathbb{Q}$ can be thought of as the minimal extension of $\mathbb{Z}$ such that every $n \in \mathbb{N}$ has an extension to a bijection $n: \mathbb{Q} \rightarrow \mathbb{Q}$.

In this paper we are interested in a general framework in which a similar extension is possible. In the construction of $\mathbb{Q}$ we make use of the fact that multiplication by a natural number is injective and commutative. It turns out that these two conditions are sufficient. More precisely, if $X$ is a nonempty set and $G$ is a commutative semigroup acting on $X$ injectively, then an extension is possible.

If $G$ fails to act injectively, then we introduce a modification of the construction so that an extension with similar properties is possible. In this case $G$ is replaced by $G^{I}$, where $I$ is an index set. If $\alpha \in G^{I}$, then $\alpha: X \rightarrow X^{I}$ and it is possible that $\alpha$ is injective even if none of the component functions is.

In the general construction no structure is assumed on $X$. If $X$ has an algebraic structure or a topology, then these induce an algebraic structure or a topology on the extended space.

In Section 3 we present some concrete interpretations of the construction in analysis.

2. Generalized Quotients. Let $X$ be a nonempty set and let $G$ be a commutative semigroup acting on $X$ injectively. This means that every $g \in G$ is an injective map $g: X \rightarrow X$ and $\left(g_{1} g_{2}\right) x=g_{1}\left(g_{2} x\right)$ for all $g_{1}, g_{2} \in G$ and $x \in X$.

Let $\mathcal{A}=X \times G$. For $(x, f),(y, g) \in \mathcal{A}$ we write

$$
(x, f) \sim(y, g) \text { if } g x=f y .
$$

It is easy to check that this is an equivalence relation in $\mathcal{A}$. Finally, we define

$$
\mathcal{B}=\mathcal{B}(X, G)=\mathcal{A} / \sim
$$

the set of generalized quotients.

The equivalence class of $(x, f)$ will be denoted by $\frac{x}{f}$. This is a slight abuse of notation, but we follow here the tradition of denoting rational numbers by $\frac{p}{q}$ even though the same formal problem is present there.

\footnotetext{
*Received May 26, 2003; accepted for publication September 4, 2003.

${ }^{\dagger}$ Department of Mathematics, University of Central Florida, Orlando, FL 32816-1364, USA (piotrm@mail.ucf.edu).
} 
Elements of $X$ can be identified with elements of $\mathcal{B}$ via the embedding $\varphi: X \rightarrow \mathcal{B}$ defined by

$$
\varphi(x)=\frac{f x}{f},
$$

where $f$ is an arbitrary element of $G$. Clearly, $\varphi$ is well-defined, that is, it is independent of $f$.

Now we consider the case when $G$ fails to act injectively on $X$. Let $I$ be a nonempty index set and let

$$
\Delta \subset G^{I}
$$

be a semigroup acting on $X$ injectively. The operation in $\Delta$ is defined in the obvious way: if $\alpha, \beta \in \Delta$, then $(\alpha \beta)(i)=\alpha(i) \beta(i), i \in I$. If $\alpha \in \Delta$, then the action of $\alpha$ on $x \in X$ is defined as $(\alpha x)(i)=\alpha(i) x$, so that

$$
\Delta \ni \alpha: X \rightarrow X^{I} .
$$

We assume that these maps are injective.

A $\Delta$ like that may not exist. An obvious necessary condition is that $G$ is a total set, i.e., if $x, y \in X$ and $x \neq y$, then there exists a $g \in G$ such that $g(x) \neq g(y)$. In what follows, we assume that a semigroup $\Delta$ with the desired property exists.

Suppose $\mathcal{X} \subset X^{I}$ satisfies the following conditions:

(a) $\alpha x \in \mathcal{X}$ for all $\alpha \in \Delta$ and all $x \in X$,

(b) $\alpha \xi \in \mathcal{X}$ for all $\alpha \in \Delta$ and all $\xi \in \mathcal{X}$.

Here, for $\xi \in \mathcal{X}$ and $\alpha \in \Delta$, we define $(\alpha \xi)(i)=\alpha(i) \xi(i), i \in I$. The above conditions are trivially satisfied if we take $\mathcal{X}=X^{I}$, but there are important applications where it is essential that $\mathcal{X}$ is a proper subset of $X^{I}$.

Let

$$
\mathcal{A}=\{(\xi, \alpha): \xi \in \mathcal{X}, \alpha \in \Delta, \text { and } \alpha(i) \xi(j)=\alpha(j) \xi(i), i, j \in I\} .
$$

Note that, by $(\mathrm{a}),(\alpha x, \alpha) \in \mathcal{A}$ for all $x \in X$ and $\alpha \in \Delta$. Moreover, if $(\xi, \alpha) \in \mathcal{A}$, then $(\beta \xi, \beta \alpha) \in \mathcal{A}$ for all $\beta \in \Delta$, by (b).

For $(\xi, \alpha),(\zeta, \beta) \in \mathcal{A}$ we write

$$
(\xi, \alpha) \sim(\zeta, \beta) \text { if } \alpha(i) \zeta(j)=\beta(j) \xi(i) \text { for all } i, j \in I
$$

It can be shown that $\sim$ is an equivalence in $\mathcal{A}$. Now we define

$$
\mathcal{B}=\mathcal{B}(\mathcal{X}, \Delta)=\mathcal{A} / \sim \text {. }
$$

As before, the equivalence class of $(\xi, \alpha)$ will be denoted by $\frac{\xi}{\alpha}$ and called a generalized quotient.

Note that the construction for the case when $G$ is a semigroup acting on $X$ injectively is a special case of the above one. Indeed, it suffices to take $I=\{1\}$, $\mathcal{X}=X$, and $\Delta=G$.

LEMma 2.1. If $(\xi, \alpha),(\zeta, \beta) \in \mathcal{A}$, then $(\xi, \alpha) \sim(\zeta, \beta)$ if and only if $\beta \xi=\alpha \zeta$.

Proof. Clearly $(\xi, \alpha) \sim(\zeta, \beta)$ implies $\beta \xi=\alpha \zeta$. Now assume $\beta \xi=\alpha \zeta$, that is,

$$
\beta(i) \xi(i)=\alpha(i) \zeta(i)
$$


for all $i \in I$. Then, for any $i, j, k \in I$ we have

$$
\beta(i) \alpha(j) \beta(k) \xi(i)=\alpha(i) \alpha(j) \beta(k) \zeta(i)
$$

and, since $(\xi, \alpha),(\zeta, \beta) \in \mathcal{A}$,

$$
\beta(i) \alpha(i) \beta(k) \xi(j)=\alpha(i) \alpha(j) \beta(i) \zeta(k) .
$$

Injectivity of $\alpha$ and $\beta$ implies

$$
\beta(k) \xi(j)=\alpha(j) \zeta(k)
$$

for all $j, k \in I$, which means $(\xi, \alpha) \sim(\zeta, \beta)$.

Note that, for any $x \in X$ and any $\alpha, \beta \in \Delta$ we have $(\alpha x, \alpha) \sim(\beta x, \beta)$. Hence elements of $X$ can be identified with elements of $\mathcal{B}$ via the embedding $\varphi: X \rightarrow \mathcal{B}$ defined by

$$
\varphi(x)=\frac{\alpha x}{\alpha},
$$

where $\alpha$ is an arbitrary element of $\Delta$.

The action of $G$ on $X$ can be extended to $\mathcal{B}$. Indeed, for any $g \in G$ and $\frac{\xi}{\alpha} \in \mathcal{B}$ we can define

$$
g \frac{\xi}{\alpha}=\frac{g \xi}{\alpha} .
$$

It is also possible to extend the action of $\Delta$ to $\mathcal{B}$ so that

$$
\gamma \frac{\xi}{\alpha}=\gamma \frac{\zeta}{\beta} \text { implies } \frac{\xi}{\alpha}=\frac{\zeta}{\beta}
$$

whenever $\gamma \in \Delta$. One should be careful with interpreting expressions of the form $\gamma \frac{\xi}{\alpha}$. If $\gamma \in \Delta$ and $\frac{\xi}{\alpha} \in \mathcal{B}$, then $\gamma \frac{\xi}{\alpha}$ is an element of $\mathcal{B}^{I}$ defined by

$$
\left(\gamma \frac{\xi}{\alpha}\right)(i)=\frac{\gamma(i) \xi}{\alpha} .
$$

Consequently,

$$
\gamma \frac{\xi}{\alpha}=\gamma \frac{\zeta}{\beta}
$$

is equivalent to

$$
\gamma(i) \beta(k) \xi(j)=\gamma(i) \alpha(j) \zeta(k)
$$

which implies $\beta(k) \xi(j)=\alpha(j) \zeta(k)$ and thus $\frac{\xi}{\alpha}=\frac{\zeta}{\beta}$.

Since $\mathcal{B}$ and $\Delta$ satisfy the conditions necessary for the construction of generalized quotients, it is natural to ask whether by repeating the construction we can obtain a still larger extension of $X$. The following two theorems address this question.

TheOrem 2.2. If $G$ acts on $X$ injectively, then $\mathcal{B}(X, G)=\mathcal{B}(\mathcal{B}(X, G), G)$. 
Proof. We show that $\Phi: \mathcal{B}(\mathcal{B}(X, G), G) \rightarrow \mathcal{B}(X, G)$ defined by

$$
\Phi\left(\frac{\frac{x}{h}}{g}\right)=\frac{x}{g h}
$$

is a bijection. Note that

$$
\frac{\frac{x_{1}}{h_{1}}}{g_{1}}=\frac{\frac{x_{2}}{h_{2}}}{g_{2}}
$$

implies $g_{2} \frac{x_{1}}{h_{1}}=g_{1} \frac{x_{2}}{h_{2}}$, and hence, $h_{2} g_{2} x_{1}=h_{1} g_{1} x_{2}$, which means $\frac{x_{1}}{g_{1} h_{1}}=\frac{x_{2}}{g_{2} h_{2}}$. Therefore $\Phi$ is well defined. Since

$$
\Phi\left(\frac{\frac{h x}{h}}{g}\right)=\frac{h x}{h g}=\frac{x}{g},
$$

$\Phi$ is surjective. Now assume

$$
\Phi\left(\frac{\frac{x_{1}}{h_{1}}}{g_{1}}\right)=\Phi\left(\frac{\frac{x_{2}}{h_{2}}}{g_{2}}\right) .
$$

Then $\frac{x_{1}}{g_{1} h_{1}}=\frac{x_{2}}{g_{2} h_{2}}$ and consequently $g_{2} \frac{x_{1}}{h_{1}}=g_{1} \frac{x_{2}}{h_{2}}$. But this means that

$$
\frac{\frac{x_{1}}{h_{1}}}{g_{1}}=\frac{\frac{x_{2}}{h_{2}}}{g_{2}},
$$

proving that $\Phi$ is injective.

ThEOREM 2.3. If for any $\alpha_{n} \in \Delta, n \in I$, there exists a $\kappa: I \rightarrow I$ such that $\left(\alpha_{n}(\kappa(n))\right) \in \Delta$, then $\mathcal{B}(\mathcal{Y}, \Delta)=\mathcal{B}(\mathcal{X}, \Delta)$ where $\mathcal{Y}=\mathcal{B}(\mathcal{X}, \Delta)^{I}$.

Proof. Suppose $\frac{F}{\beta} \in \mathcal{B}(\mathcal{Y}, \Delta)$, where $F(n)=\frac{\xi_{n}}{\alpha_{n}}$ and $\alpha_{n}, \beta \in \Delta$. Then

$$
\beta(n) F(m)=\beta(m) F(n)
$$

for all $m, n \in I$. Hence, for all $i, j, m, n \in I$,

$$
\beta(n) \alpha_{n}(i) \alpha_{m}(j) F(m)=\beta(m) \alpha_{m}(j) \alpha_{n}(i) F(n)
$$

and

$$
\beta(n) \alpha_{n}(i) \xi_{m}(j)=\beta(m) \alpha_{m}(j) \xi_{n}(i),
$$

since $\alpha_{k}(l) F(k)=\xi_{k}(l)$ for any $k, l \in I$. Let $\kappa: I \rightarrow I$ be such that $\left(\alpha_{n}(\kappa(n))\right) \in \Delta$. If we take $i=\kappa(n)$ and $j=\kappa(m)$ in (2.1), we obtain

$$
\beta(n) \alpha_{n}(\kappa(n)) \xi_{m}(\kappa(m))=\beta(m) \alpha_{m}(\kappa(m)) \xi_{n}(\kappa(n)),
$$

for all $m, n \in I$, which means that

$$
\frac{\xi_{n}(\kappa(n))}{\beta(n) \alpha_{n}(\kappa(n))} \in \mathcal{B}(\mathcal{X}, \Delta) .
$$


We will show that the map $\Phi: \mathcal{B}(\mathcal{Y}, \Delta) \rightarrow \mathcal{B}(\mathcal{X}, \Delta)$ defined by

$$
\Phi\left(\frac{F}{\beta}\right)=\frac{\xi_{n}(\kappa(n))}{\beta(n) \alpha_{n}(\kappa(n))}
$$

is a bijection.

First note that (2.1) implies that $\Phi$ is independent of a particular $\kappa$. If $F(n)=\frac{\xi_{n}}{\alpha_{n}}$, $G(n)=\frac{\zeta_{n}}{\delta_{n}}$, and $\frac{F}{\beta}=\frac{G}{\gamma}$, then

$$
\gamma(m) \delta_{m}(i) \alpha_{n}(j) F(n)=\beta(n) \alpha_{n}(j) \delta_{m}(i) G(m)
$$

for all $i, j, m, n \in I$. But this means that

$$
\gamma(m) \delta_{m}(i) \xi_{n}(j)=\beta(n) \alpha_{n}(j) \zeta_{m}(i)
$$

for all $i, j, m, n \in I$. Let $\iota, \kappa: I \rightarrow I$ be such that $\left(\alpha_{n}(\iota(n))\right),\left(\delta_{n}(\kappa(n))\right) \in \Delta$. From (2.2) we obtain

$$
\gamma(m) \delta_{m}(\kappa(m)) \xi_{n}(\iota(n))=\beta(n) \alpha_{n}(\iota(n)) \zeta_{m}(\kappa(m))
$$

for all $m, n \in I$, which implies

$$
\frac{\xi_{n}(\iota(n))}{\beta(n) \alpha_{n}(\iota(n))}=\frac{\zeta_{n}(\kappa(n))}{\gamma(n) \delta_{n}(\kappa(n))} .
$$

Therefore $\Phi$ is well-defined.

Now take $F(n)=\frac{\xi_{n}}{\alpha_{n}}$ and $G(n)=\frac{\zeta_{n}}{\delta_{n}}$ and assume that

$$
\Phi\left(\frac{F}{\beta}\right)=\Phi\left(\frac{G}{\gamma}\right) .
$$

Then

$$
\frac{\xi_{n}(\iota(n))}{\beta(n) \alpha_{n}(\iota(n))}=\frac{\zeta_{n}(\kappa(n))}{\gamma(n) \delta_{n}(\kappa(n))},
$$

for some $\iota, \kappa: I \rightarrow I$. Hence, for all $m, n \in I$, we have

$$
\gamma(m) \delta_{m}(\kappa(m)) \xi_{n}(\iota(n))=\beta(n) \alpha_{n}(\iota(n)) \zeta_{m}(\kappa(m))
$$

and

$$
\gamma(m) \delta_{m}(\kappa(m)) \alpha_{n}(\iota(n)) F(n)=\beta(n) \alpha_{n}(\iota(n)) \delta_{m}(\kappa(m)) G(m) .
$$

Since $\left(\alpha_{i}(\iota(i))\right),\left(\delta_{i}(\kappa(i))\right) \in \Delta$, we obtain

$$
\gamma(m) F(n)=\beta(n) G(m),
$$

which means that $\frac{F}{\beta}=\frac{G}{\gamma}$. This proves that $\Phi$ is injective. Finally, for any $\frac{\xi}{\alpha} \in \mathcal{B}$ we have

$$
\Phi\left(\frac{\frac{\beta(n) \xi}{\alpha}}{\beta(n)}\right)=\frac{\beta(n) \xi(n)}{\beta(n) \alpha(n)}=\frac{\xi(n)}{\alpha(n)}
$$


which proves that $\Phi$ is surjective.

The condition in the above theorem is satisfied in Examples 3.5 and 3.6 in the next section. It is clear that it is always satisfied if $I=\mathbb{N}$ and $\Delta$ is defined as

$$
\Delta=\left\{\alpha \in X^{I}: \Lambda(\alpha(i)) \rightarrow 0 \text { as } i \rightarrow \infty\right\}
$$

for some $\Lambda: X \rightarrow[0, \infty)$. More examples of this kind can be found in [6]. In Examples 3.4 and 3.7 the condition is not satisfied, but the conclusion of the theorem remains true in both cases (see [3] for more on Example 3.4 and [5] and [6] for Example 3.7). The author is not aware of an example for which reapplication of the construction produces an essentially larger space.

Now we assume that $X$ is a multiplicative group and $G$ is a semigroup of injective commuting homomorphisms from $X$ to $X$. For $\frac{x}{f}, \frac{y}{g} \in \mathcal{B}$ define

$$
\frac{x}{f} \frac{y}{g}=\frac{g(x) f(y)}{f g} .
$$

It is easy to check that this is a well-defined group operation in $\mathcal{B}$. If $1_{X}$ is the identity element in $X$, then $\frac{f 1_{X}}{f}=\frac{1_{X}}{f}$ is the identity element in $\mathcal{B}$. Moreover, the embedding $\varphi: X \rightarrow \mathcal{B}$ defined by $\varphi(x)=\frac{f x}{f}$ is a group homomorphism.

The same idea works in the general case: for $\frac{\xi}{\alpha}, \frac{\zeta}{\beta} \in \mathcal{B}$, we define

$$
\frac{\xi}{\alpha} \frac{\zeta}{\beta}=\frac{\beta(\xi) \alpha(\zeta)}{\alpha \beta} .
$$

Then $\mathcal{B}$ becomes a group and the embedding $\varphi: X \rightarrow \mathcal{B}$ defined by $\varphi(x)=\frac{\alpha x}{\alpha}$ is a group homomorphism.

It is easy to see that, if $X$ is Abelian, so is $\mathcal{B}$. More generally, if $X$ is a $\Lambda$-module such that

$$
g(x+y)=g x+g y \text { and } g(\lambda x)=\lambda(g x),
$$

for all $x, y \in X, g \in G$, and $\lambda \in \Lambda$, then $\mathcal{B}$ is a $\Lambda$-module with

$$
\frac{\xi}{\alpha}+\frac{\zeta}{\beta}=\frac{\beta \xi+\alpha \zeta}{\alpha \beta} \text { and } \quad \lambda \frac{\xi}{\alpha}=\frac{\lambda \xi}{\alpha}
$$

and $\varphi$ is an isomorphism of $X$ with a submodule of $\mathcal{B}$.

Now let $X$ be a topological space and let $G$ be a commutative semigroup of continuous maps acting on $X$, equipped with a topology. On $\mathcal{X}$ and $\Delta$ we put the topologies induced by the product topologies on $X^{I}$ and $G^{I}$, respectively. The topology of $\mathcal{A}$ is induced by the product topology on $\mathcal{X} \times \Delta$. Finally, we put the quotient topology on $\mathcal{B}$.

TheOREM 2.4. The embedding $\varphi: X \rightarrow \mathcal{B}$ is continuous.

Proof. Fix an $\alpha \in \Delta$. Then $\varphi$ is the composition of two continuous maps: the map $x \mapsto(\alpha x, \alpha)$ and the quotient map $(\alpha x, \alpha) \mapsto\left[\frac{\alpha x}{\alpha}\right]$. 
3. Examples. Concrete realizations of the construction produce familiar objects and create some new possibilities.

EXAMPLE 3.1. If $X$ is an integral domain and

$$
G=\{x \in X: x \neq 0\},
$$

then $G$ acts on $X$ injectively and $\mathcal{B}$ is the field of quotients.

EXAmple 3.2. Let $X$ be a nonempty set with an injection $g: X \rightarrow X$ and let $G=\left\{1_{X}, g, g^{2}, g^{3}, \ldots\right\}$. Then $\mathcal{B}$ is the minimal extension of $X$ such that $g^{-n}: \mathcal{B} \rightarrow \mathcal{B}$ is well defined for all $n \in \mathbb{N}$.

For example, let $X=\mathbb{Z}$. If $g: \mathbb{Z} \rightarrow \mathbb{Z}$ is defined by $g x=2 x$, then $\mathcal{B}$ is the set of all rational numbers of the form $\frac{p}{2^{n}}$. This is the minimal set that contains all integers and such that division by 2 is always possible.

EXAmPle 3.3. Let $X=\mathcal{C}\left(\mathbb{R}^{N}\right)$, the space of all continuous functions on $\mathbb{R}^{N}$, and let

$$
\left(\Lambda_{k} x\right)\left(t_{1}, \ldots, t_{N}\right)=\int_{0}^{t_{k}} x\left(t_{1}, \ldots, s_{k}, \ldots, t_{N}\right) d s_{k},
$$

for $k=1, \ldots, N$. Note that these maps are injective. Let $G$ be the semigroup generated by $\Lambda_{1}, \ldots, \Lambda_{N}$. Since $\Lambda_{k}^{-1}$ corresponds to $\frac{\partial}{\partial t_{k}}, \mathcal{B}$ is the minimal extension of $\mathcal{C}\left(\mathbb{R}^{N}\right)$ where differentiation is always possible. This space $\mathcal{B}$ can be identified with the space of all distributions of finite order on $\mathbb{R}^{N}$. The action of $\left[\frac{x}{\Lambda_{1}^{k_{1}} \ldots \Lambda_{N}^{k_{N}}}\right] \in \mathcal{B}$ on a test function $\varphi$ can be defined by

$$
\left\langle\frac{x}{\Lambda_{1}^{k_{1}} \ldots \Lambda_{N}^{k_{N}}}, \varphi\right\rangle=(-1)^{k_{1}+\cdots+k_{N}} \int_{\mathbb{R}^{N}} x(t) \frac{\partial^{k_{1}+\cdots+k_{N}}}{\partial t_{1}^{k_{1}} \ldots \partial t_{N}^{k_{N}}} \varphi(t) d t,
$$

where $t=\left(t_{1}, \ldots, t_{N}\right)$.

A function $x \in \mathcal{C}\left(\mathbb{R}^{N}\right)$ is called slowly increasing if

$$
\left|x\left(t_{1}, \ldots, t_{N}\right)\right| \leq C\left(1+t_{1}^{2}+\cdots+t_{N}^{2}\right)^{n}
$$

for some $C>0$ and $n \in \mathbb{N}$ and all $\left(t_{1}, \ldots, t_{N}\right) \in \mathbb{R}^{N}$. If we replace $\mathcal{C}\left(\mathbb{R}^{N}\right)$ by the space of slowly increasing functions and use the same $G$, then the obtained $\mathcal{B}$ can be identified with the space of tempered distributions, again by (3.1).

EXAmPLE 3.4. Let $X=\mathcal{C}(\mathbb{R})$ and let $G$ be the convolution semigroup generated by characteristic functions of bounded intervals. An element of $G$ can be written in the form $\varphi_{1} * \varphi_{2} * \cdots * \varphi_{m}$, where $\varphi_{1}, \varphi_{2}, \ldots, \varphi_{m}$ are characteristic functions of bounded intervals in $\mathbb{R}$. We let $G$ act on $X$ by convolution. Note that the mapping $x \mapsto \varphi * x$ is not injective.

Let $\omega$ be the characteristic function of the interval $\left[-\frac{1}{2}, \frac{1}{2}\right]$. For $n \in \mathbb{N}$, let $\omega^{n}$ denote the convolution of $n$ copies of $\omega$, i.e., $\omega^{1}=\omega$ and $\omega^{n}=\omega * \omega^{n-1}$ for $n>1$. Let $I=(0, \infty)$,

$$
\Delta=\left\{\varphi: \varphi(\varepsilon)(t)=\varepsilon \psi(\varepsilon t) \text { where } \psi=\omega^{m}, m \in \mathbb{N}, \varepsilon \in I\right\}
$$


and $\mathcal{X}=X^{I}$. It is not as obvious as in Example 3.3, but it can be shown that in this case $\mathcal{B}$ can be identified with the space of all distributions of finite order on $\mathbb{R}$ (see [3]). If we replace $\mathcal{C}(\mathbb{R})$ by the space of continuous functions with compact support, then the obtained $\mathcal{B}$ can be identified with the space of distribution with compact support. It is not clear if the construction in Example 3.3 can be modified to produce distributions with compact support.

EXAMPLE 3.5. Consider a sequence of spaces

$$
\cdots \subset X_{-2} \subset X_{-1} \subset X_{0} \subset X_{1} \subset X_{2} \subset \cdots
$$

with commuting projections

$$
\pi_{n}: \bigcup_{k \in \mathbb{Z}} X_{k} \rightarrow X_{n}
$$

Let

$$
X=\bigcup_{k \in \mathbb{Z}} X_{k}, G=\left\{\pi_{n}: n \in \mathbb{Z}\right\}
$$

and

$$
\Delta=\left\{\left(\pi_{k_{n}}\right): k_{n} \rightarrow \infty\right\}, \mathcal{X}=X^{I} .
$$

The obtained space $\mathcal{B}$ consists of objects that can be projected on every $X_{n}$ and are determined by these projections.

An important example of the above is multiresolution analysis. Let $\varphi \in L^{2}(\mathbb{R})$. For $m, n \in \mathbb{Z}$ define

$$
\varphi_{m, n}(t)=2^{m / 2} \varphi\left(2^{m} t-n\right) .
$$

Assume that $\varphi$ is such that $\left\{\varphi_{0, n}: n \in \mathbb{Z}\right\}$ is an orthonormal set in $L^{2}(\mathbb{R})$ and define

$$
X_{m}=\left\{\sum_{n \in \mathbb{Z}} c_{n} \varphi_{m, n}:\left(c_{n}\right) \in l^{2}(\mathbb{Z})\right\} .
$$

Then $X_{n} \subset L^{2}(\mathbb{R})$ for all $n \in \mathbb{Z}$ and $X_{m} \subset X_{n}$ when $m<n$. Let $X=\bigcup_{m \in \mathbb{Z}} X_{m}$ and let $\pi_{m}$ denotes the orthogonal projection on $X_{m}$.

EXAMPLE 3.6. In [2] an example of $\mathcal{B}$ isomorphic to the set of all positive Radon measures on $\mathbb{R}^{N}$ is given. Here we present a modification of that construction that produces all Radon measures on $\mathbb{R}^{N}$.

Let

$$
I_{\bar{i}, k}=\left[\frac{i_{1}}{2^{k}}, \frac{i_{1}+1}{2^{k}}\right) \times \cdots \times\left[\frac{i_{N}}{2^{k}}, \frac{i_{N}+1}{2^{k}}\right) \subset \mathbb{R}^{N},
$$

for $\bar{i}=\left(i_{1}, i_{2}, \ldots, i_{N}\right) \in \mathbb{Z}^{N}$ and $k \in \mathbb{N}_{0}=\{0,1,2, \ldots\}$, and let $\Omega(\bar{i}, k)$ denote the characteristic function of $\bar{I}_{\bar{i}, k}$. For $k \in \mathbb{N}_{0}$ we define

$$
X_{k}=\left\{\sum_{\bar{i} \in \mathbb{Z}^{N}} \alpha_{\bar{i}} \Omega(\bar{i}, k): \bar{i} \in \mathbb{Z}^{N}\right\} \quad \text { and } \quad X=\bigcup_{k \in \mathbb{N}_{0}} X_{k} .
$$


For $k \in \mathbb{N}_{0}$, let $A_{k}: X \rightarrow X_{k}$ be the projection defined by

$$
A_{k} f=\sum_{\bar{i} \in \mathbb{Z}^{N}}\left(2^{N k} \int_{I_{\bar{i}, k}} f\right) \Omega(\bar{i}, k),
$$

and let

$$
G=\left\{A_{0}, A_{1}, A_{2}, \ldots\right\} .
$$

Then $G$ is a commutative semigroup acting on $X$. We take $I=\mathbb{N}_{0}$ and let

$$
\Delta=\left\{\left(A_{p_{k}}\right): p_{k} \rightarrow \infty\right\} .
$$

Finally, let

$$
\mathcal{X}=\left\{\left(f_{n}\right) \in X^{I}: A_{0}\left|f_{n}\right| \leq C \text { for some } C \text { and all } n \in \mathbb{N}\right\} .
$$

Note that $\mathcal{X}$ satisfies (a) and (b). We will show that in this case $\mathcal{B}$ is the collection of all Radon measures on $\mathbb{R}^{N}$.

Let $\mathcal{K}$ be the space of all real-valued continuous functions on $\mathbb{R}^{N}$ with compact support. A sequence of functions $\varphi_{n} \in \mathcal{K}$ converges in $\mathcal{K}$ if it converges uniformly and $\operatorname{supp} \varphi_{n} \subset K$ for some compact $K$ and all $n \in \mathbb{N}$. Note that (3.2) can be used to define mappings $A_{k}: \mathcal{K} \rightarrow X_{k}$.

Consider $\frac{f_{n}}{A_{p_{n}}} \in \mathcal{B}$ and $\varphi \in \mathcal{K}$. Let $\varepsilon$ be a positive number. Since $\varphi$ is uniformly continuous, there exists an $l \in \mathbb{N}_{0}$ such that

$$
\left\|\varphi-A_{p_{l}} \varphi\right\|_{\infty}<\varepsilon .
$$

For any $m, n \in \mathbb{N}$ we have

$$
\left|\int f_{m} \varphi-\int f_{n} \varphi\right| \leq \int\left|f_{m}-f_{n}\right|\left|\varphi-A_{p_{l}} \varphi\right|+\left|\int\left(f_{m}-f_{n}\right) A_{p_{l}} \varphi\right|,
$$

and

$$
\begin{aligned}
\int\left(f_{m}-f_{n}\right) A_{p_{l}} \varphi & =\int\left(A_{p_{l}} f_{m}-A_{p_{l}} f_{n}\right) A_{p_{l}} \varphi \\
& =\int\left(A_{m} f_{p_{l}}-A_{n} f_{p_{l}}\right) A_{p_{l}} \varphi=0
\end{aligned}
$$

for all sufficiently large $m$ and $n$. Let $E$ be a finite union of $I_{\bar{i}, 0}$ 's containing the support of $\varphi$. Then

$$
\begin{aligned}
\int\left|f_{m}-f_{n}\right|\left|\varphi-A_{p_{l}} \varphi\right| & \leq \varepsilon \int_{E}\left|f_{m}-f_{n}\right| \\
& \leq \varepsilon \int_{E}\left(\left|f_{m}\right|+\left|f_{n}\right|\right) \\
& =\varepsilon \int_{E}\left(A_{0}\left|f_{m}\right|+A_{0}\left|f_{n}\right|\right) \leq \varepsilon c,
\end{aligned}
$$

where $c$ is a constant that is independent of $m$ and $n$. This shows that $\left(\int f_{n} \varphi\right)$ is a Cauchy sequence. Moreover, different representatives of $F$ produce equivalent Cauchy sequences. Consequently, every $F=\frac{f_{n}}{A_{p_{n}}} \in \mathcal{B}$ defines a functional on $\mathcal{K}$ via

$$
\Lambda_{F}(\varphi)=\lim _{n \rightarrow \infty} \int f_{n} \varphi
$$


The functional is obviously linear. Since,

$$
\left|\int f_{n} \varphi\right| \leq\|\varphi\|_{\infty} \int_{E} A_{0}\left|f_{n}\right| \leq C \lambda(E)\|\varphi\|_{\infty}
$$

where $\lambda(E)$ is the Lebesgue measure of $E$ and $C$ is a constant independent of $n$, we have

$$
\left|\Lambda_{F}(\varphi)\right| \leq C \lambda(E)\|\varphi\|_{\infty}
$$

Thus $\Lambda_{F}$ is a bounded linear functional on $\mathcal{K}$ and, by the Riesz Representation Theorem, there exists a Radon measure $\mu$ such that $\Lambda_{F}(\varphi)=\int \varphi d \mu$ for all $\varphi \in \mathcal{K}$.

Now assume that $\mu$ is a Radon measure on $\mathbb{R}^{N}$. Define

$$
f_{n}=\sum_{\bar{i} \in \mathbb{Z}^{N}} 2^{N n} \mu\left(I_{\bar{i}, n}\right) \Omega(\bar{i}, n) .
$$

Then $F=\frac{f_{n}}{A_{n}} \in \mathcal{B}$. Moreover, the Radon measure corresponding to $\Lambda_{F}$ is $\mu$.

EXAMPLE 3.7. In this example we recall the construction of Boehmians (see, for example, [5] or [7]). This example is what motivated the construction discussed in this paper.

Let $X=\mathcal{C}\left(\mathbb{R}^{N}\right)$ and let $G=\mathcal{D}$, the space of all compactly supported smooth functions on $\mathbb{R}^{N}$, acting on $X$ by convolution. Let $I=\mathbb{N}$,

$$
\Delta=\left\{\left(\varphi_{n}\right) \in \mathcal{D}^{\mathbb{N}}: \varphi_{n}(x)=n^{N} \psi(n x) \text { where } \psi \in \mathcal{D} \text { and } \int \psi=1\right\}
$$

and $\mathcal{X}=X^{I}$. The obtained $\mathcal{B}$ is a space of generalized functions containing all Schwartz distributions as a proper subspace. One can use other function spaces and different sets $\Delta$ and obtain different spaces of generalized functions, sharing the common name of Boehmians. For examples of such spaces of Boehmians see [1], [4], [8], [9]. An extensive list of papers on Boehmians can be found at http://math.ucf.edu/ piotr/boehmians.html.

\section{REFERENCES}

[1] J. J. Betancor, M. Linares, And J. M. R. MÉndez, Ultraspherical transform of summable Boehmians, Math. Japon., 44 (1996), pp. 81-88.

[2] J. Burzyk AND P. Mikusiński, A generalization of the construction of a field of quotients with applications in analysis, to appear.

[3] J. BurzyK AND P. Mikusiński, A characterization of distribution of finite order in the space of Boehmians, Integral Transform. Spec. Funct., to appear.

[4] V. Karunakaran and R. Roopkumar, Ultra Boehmians and their Fourier transforms, Fract. Calc. Appl. Anal., 5 (2002), pp. 181-194.

[5] P. Mikusiński, Convergence of Bohmians, Japan. J. Math., 9 (1983), pp. 159-179.

[6] P. Mikusiński, On delta sequences, Rend. Istit. Mat. Univ. Trieste, 19 (1987), pp. 165-175.

[7] P. Mikusiński, Boehmians and Generalized Functions, Acta Math. Hung., 51 (1988), pp. $271-281$.

[8] P. Mikusinski And M. Morimoto, Boehmians on the sphere and their spherical harmonic expansions, Fract. Calc. Appl. Anal., 4 (2001), pp. 25-35.

[9] D. Nemzer, Periodic Boehmians. II, Bull. Austral. Math. Soc., 44 (1991), pp. 271-278. 